\section{Lung Ultrasound in COVID-19}

Chest $X$-ray is considered less sensitive in diagnosing coronavirus disease 2019 (COVID-19) pneumonia, and due to the milder disease and relatively subtle findings [1], the sensitivity may be further lowered in children. Though chest computerized tomography (CT) is the imaging modality of choice in COVID-19, difficulties in sparing a dedicated machine, transferring potentially infectious and sick patients to the $\mathrm{CT}$ room, disinfection of machine, and ionizing radiation exposure make it less appealing, especially in children. Therefore, a readily available, point-of-care tool that avoids radiation exposure is needed.

Lung ultrasound (LUS) is routinely used as a point-of-care imaging tool in emergency and intensive care units, and its role in COVID-19 is being explored. COVID-19 classically presents as diffuse bilateral pneumonia with asymmetric patchy lesions in the lung periphery that are amenable to ultrasound visualization [2]. Its easy availability, easy decontamination, freedom from radiation, and portability favor its use in COVID-19.

All 14 lung areas (three posterior, two lateral, and two anterior) should be scanned. B-lines are the most classical findings of COVID-19 pneumonia and some authors describe COVID-19 pneumonia as a "storm of clusters of B-lines", sometimes appearing as shining white lung [2]. In a metaanalysis of seven studies (122 patients), almost all patients had abnormal LUS [3]. The common abnormalities were interstitial involvement/B-pattern $(97 \%)$, pleural line abnormalities (70\%), pleural thickening (54\%), consolidation $(39 \%)$, and pleural effusion (14\%) [3]. The number and appearance of B-lines also correlate with the disease severity. As disease progresses, the B-lines increase in number and become more confluent. In severe disease, extensive areas of subpleural consolidations and pleural effusion may be visualized. On serial monitoring, a decrease in the B-lines and appearance of A-lines indicate recovery [4].
Till now only three studies ( 23 patients) have evaluated the role of LUS in pediatric patients. Most common findings were pulmonary interstitial syndrome (82\%) followed by consolidation. Only one study (5 patients) directly compared chest CT, $X$-ray, and LUS and found that ultrasound fares better than chest $X$-ray [5].

Few important limitations of LUS are the inability to detect deep and intrapulmonary lesions, difficult to scan posterobasal regions in sick patients, and relatively lower sensitivity than CT scan. Point of care lung ultrasound, where available, may be utilized in the management of children with COVID-19.

Published online: July 24, 2020; PII: S097475591600215

ARUSHI YADAV ${ }^{1}$ AND JOGENDER KUMAR ${ }^{2}$ *

${ }^{1}$ Department of Radiodiagnosis, Government Medical College and Hospital; and ${ }^{2}$ Department of Pediatrics, Post Graduate Institute of Medical Education and Research; Chandigah, India.

\section{REFERENCES}

*jogendrayadv@gmail.com

1. Balasubramanian S, Rao NM, Goenka A, Roderick M, Ramanan AV. Coronavirus disease 2019 (COVID-19) in children - What we know so far and what we do not. Indian Pediatr. 2020;57:435-42.

2. Volpicelli G, Gargani L. Sonographic signs and patterns of COVID-19 pneumonia. Ultrasound J. 2020;12:22.

3. Mohamed MFH, Al-Shokri S, Yousaf Z, Danjuma M, Parambil J, Mohamed S, et al. Frequency of abnormalities detected by point-of-care lung ultrasound in symptomatic COVID-19 patients: Systematic review and meta-analysis. Am J Trop Med Hyg. 2020. Available from: http:// www.ajtmh.org/content/journals/10.4269/ajtmh.20-0371. Accessed June 17, 2020.

4. Fiala MJ. Ultrasound in COVID-19: A timeline of ultrasound findings in relation to CT. Clin Radiol. 2020;75:553-4.

5. Feng XY, Tao XW, Zeng LK, Wang WQ, Li G. Application of pulmonary ultrasound in the diagnosis of COVID-19 pneumonia in neonates. Zhonghua $\mathrm{Er} \mathrm{Ke} \mathrm{Za} \mathrm{Zhi.}$ 2020;58:347-50.

\section{Pubertal Menorrhagia-A Rare Presentation of Congenital Factor XIII Deficiency}

Factor XIII deficiency, with an estimated incidence of 1 in 1-2 million, is an extremely rare congenital bleeding disorder with autosomal recessive inheritance and often with a history of consanguinity [1]. It can present with myriad bleeding manifestations in different age groups, with varying severity. This is partly due to multiple possible mutations of the two subunits of Factor XIII (subunit A mutations are associated with severe manifestations than those of subunit B) and partly because factor XIII is a multifunctional protein (required in wound healing, maintenance of pregnancy, angiogenesis and hemostasis). Umbilical cord bleeding, present in $73 \%$ of patients, is highly suggestive of this condition [1,2]. Pubertal menorrhagia was present in nearly $31 \%$ patients as per an Indian study [1].

A14-year-old girl presented with severe anemia and 